\title{
Supporting Information for Efficient Construction of Excited-State Hessian Matrices with Machine Learning Accelerated Multi-Layer Energy-Based Fragment Method
}

Wen-Kai Chen ${ }^{1}$, Yaolong Zhang², Bin Jiang², Wei-Hai Fang1, and Ganglong Cui ${ }^{1, *}$

${ }^{1}$ Key Laboratory of Theoretical and Computational Photochemistry, Ministry of Education, College of Chemistry, Beijing Normal University, Beijing 100875, China and ${ }^{2}$ Hefei National Laboratory for Physical Science at the Microscale, Department of Chemical Physics, Key Laboratory of Surface and Interface Chemistry and Energy Catalysis of Anhui Higher Education Institutes, University of Science and Technology of China, Hefei, Anhui 230026, China

Email: ganglong.cui@bnu.edu.cn

\section{Content}

Supporting Information for Efficient Construction of Excited-State Hessian Matrices with Machine Learning Accelerated Multi-Layer Energy-Based Fragment Method ......................................................... 1

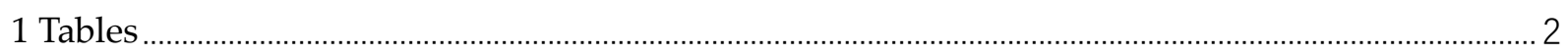

2 Figures 


\section{Tables}

Table S1: RMSD Values of Different MLEBF Methods Relative to Full QM Methods (in eV/ $\AA^{2}$ ) at Three-Body Interaction Level with Electrostatically Embedding Potentials (without in Parentheses). MLEBF(TDDFT:DFT) is Actually MLEBF(DFT:DFT) for the So State.

\begin{tabular}{|c|c|c|c|}
\hline Methods/States & $\mathrm{S}_{1}$ & $\mathrm{~S}_{0}$ & $\mathrm{~T}_{1}$ \\
\hline MLEBF(TDDFT:DFT) & $0.0133(0.0230)$ & $0.0101(0.0132)$ & $0.0103(0.0158)$ \\
\hline MLEBF(CASSCF:HF) & $0.0066(0.0082)$ & $0.0070(0.0077)$ & $0.0070(0.0111)$ \\
\hline MLEBF(CASSCF:DFT) & $0.0069(0.0093)$ & $0.0072(0.0091)$ & $0.0073(0.0118)$ \\
\hline
\end{tabular}

\section{Figures}
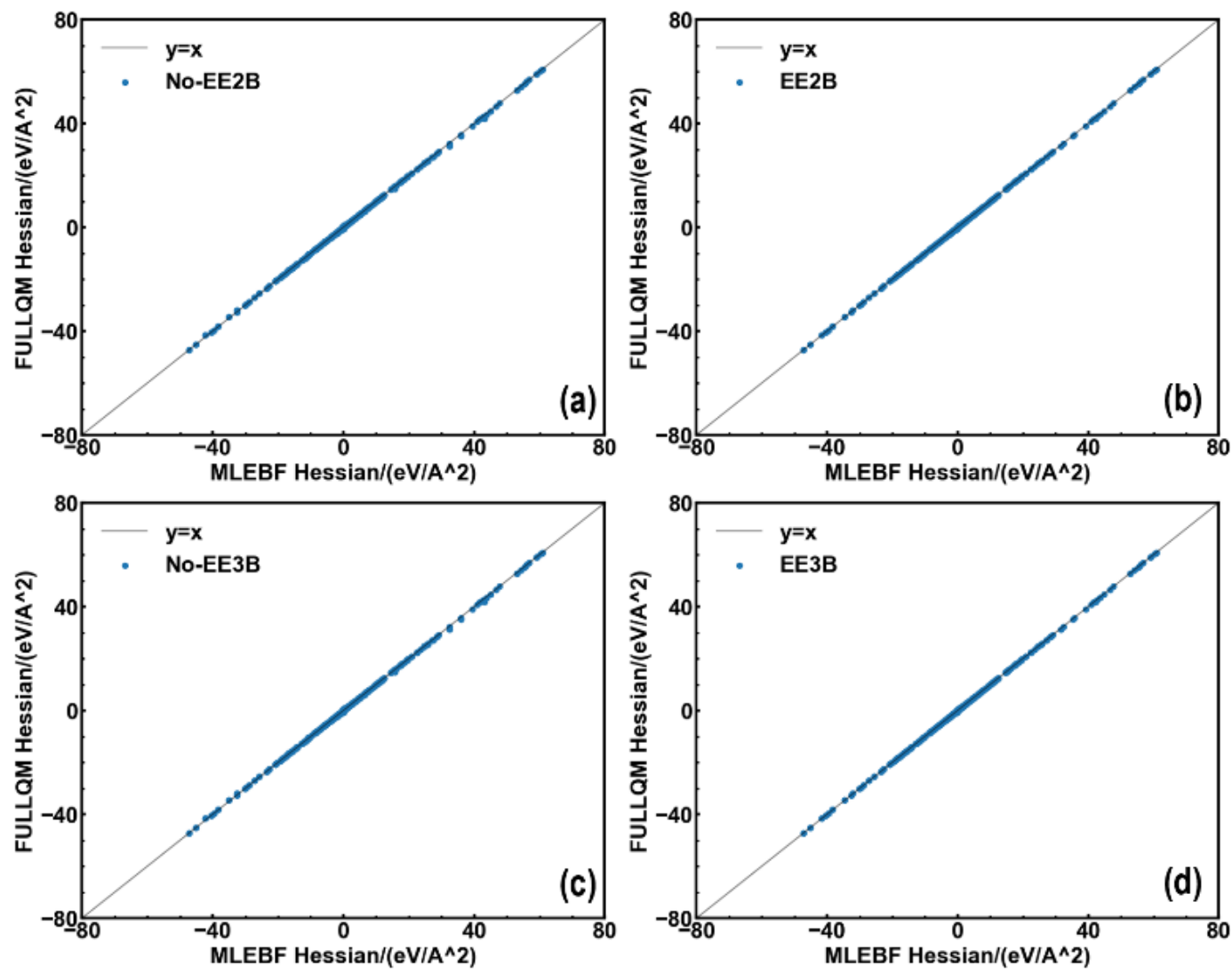

Figure S1: Comparison of full DFT and MLEBF(DFT:DFT) calculated $\mathrm{S}_{0}$ hessian matrix elements (in eV/ $/ \AA^{2}$ ) at (a-b) two- and (c-d) three-body interaction levels (a and c) without and (b and d) with electrostatically embedding potentials. 

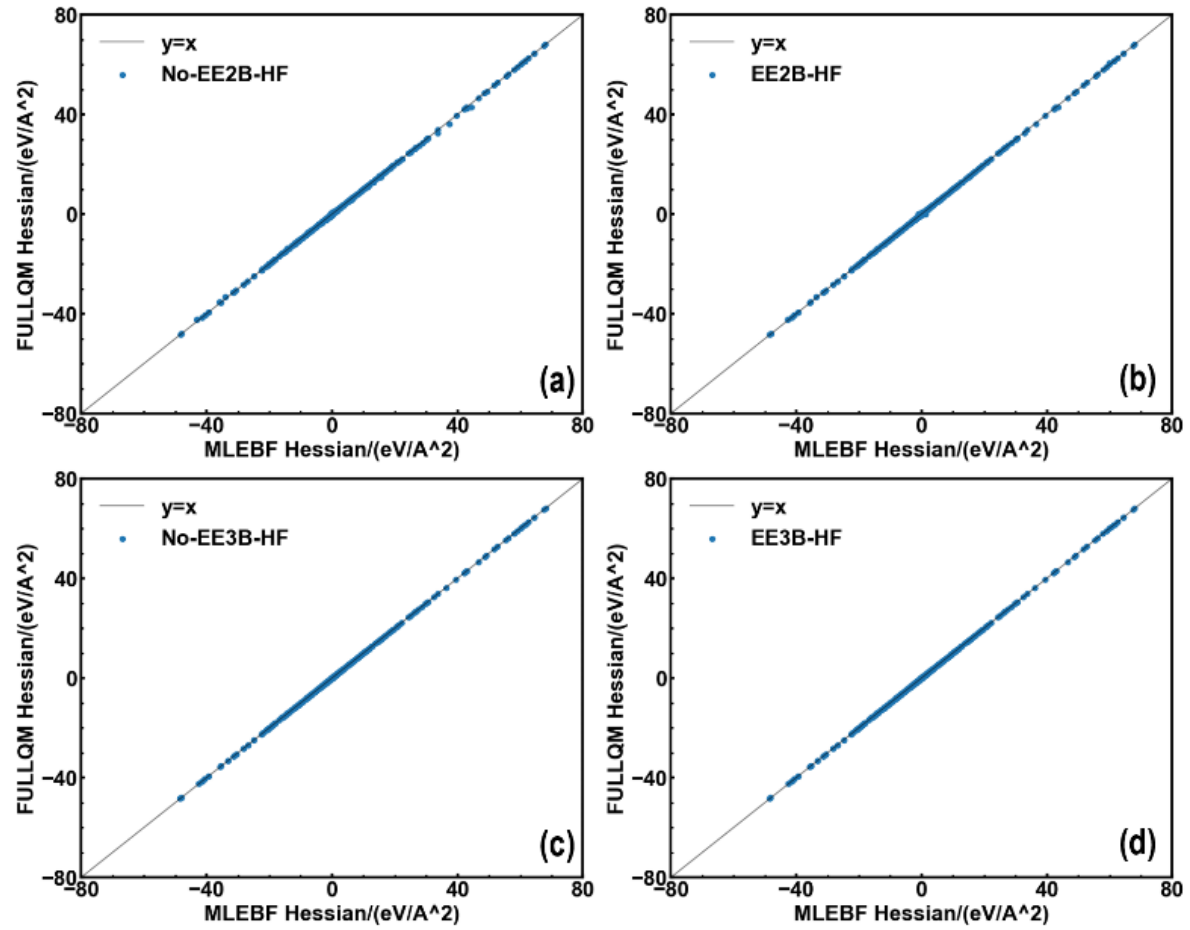

Figure S2: Comparison of full CASSCF and MLEBF(CASSCF:HF) calculated So hessian matrix elements (in eV/ $/ \AA^{2}$ ) at (a-b) two- and (c-d) three-body interaction levels (a and c) without and $(b$ and $d)$ with electrostatically embedding potentials.
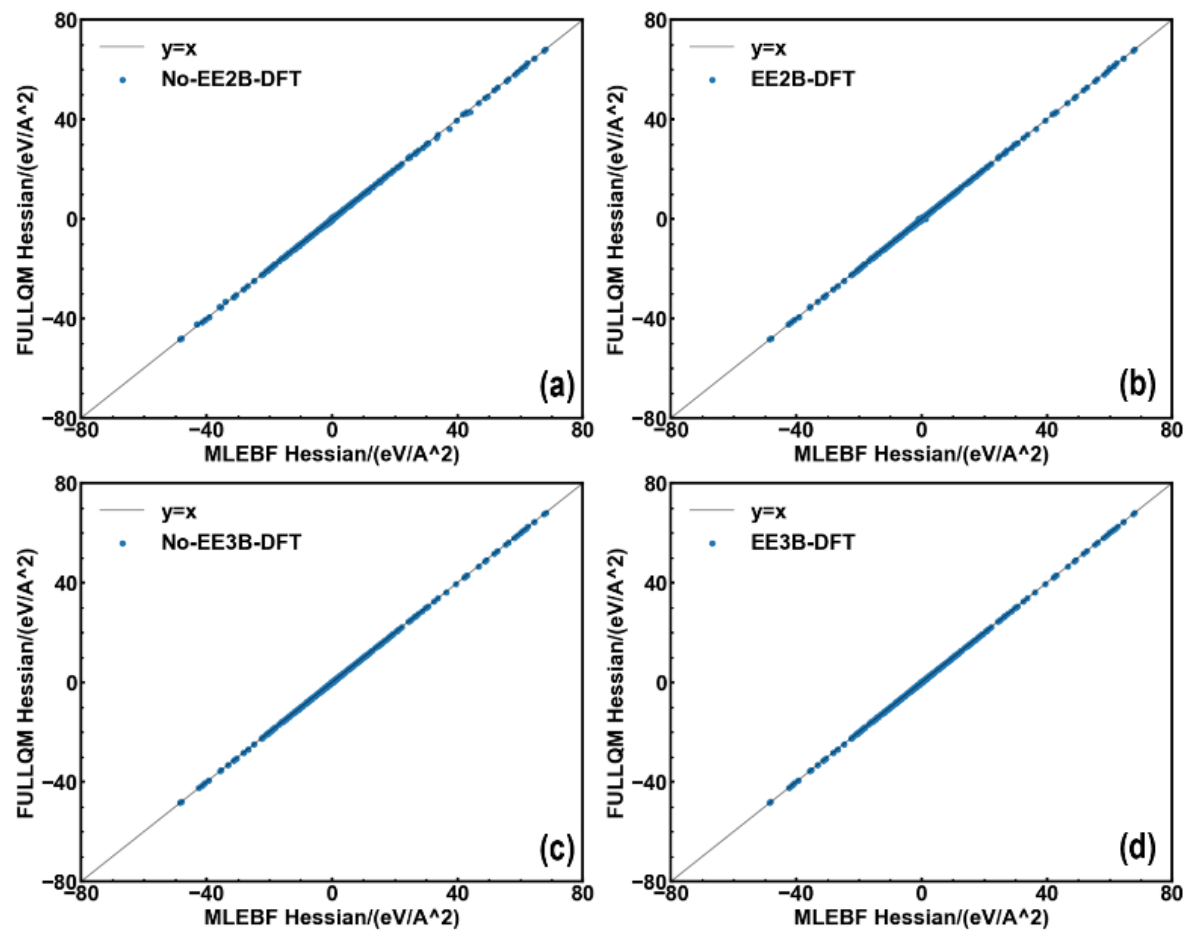

Figure S3: Comparison of full CASSCF and MLEBF(CASSCF:DFT) calculated So hessian matrix elements (in eV/ $\AA^{2}$ ) at (a-b) two- and (c-d) three-body interaction levels (a and c) without and $(b$ and $d)$ with electrostatically embedding potentials. 

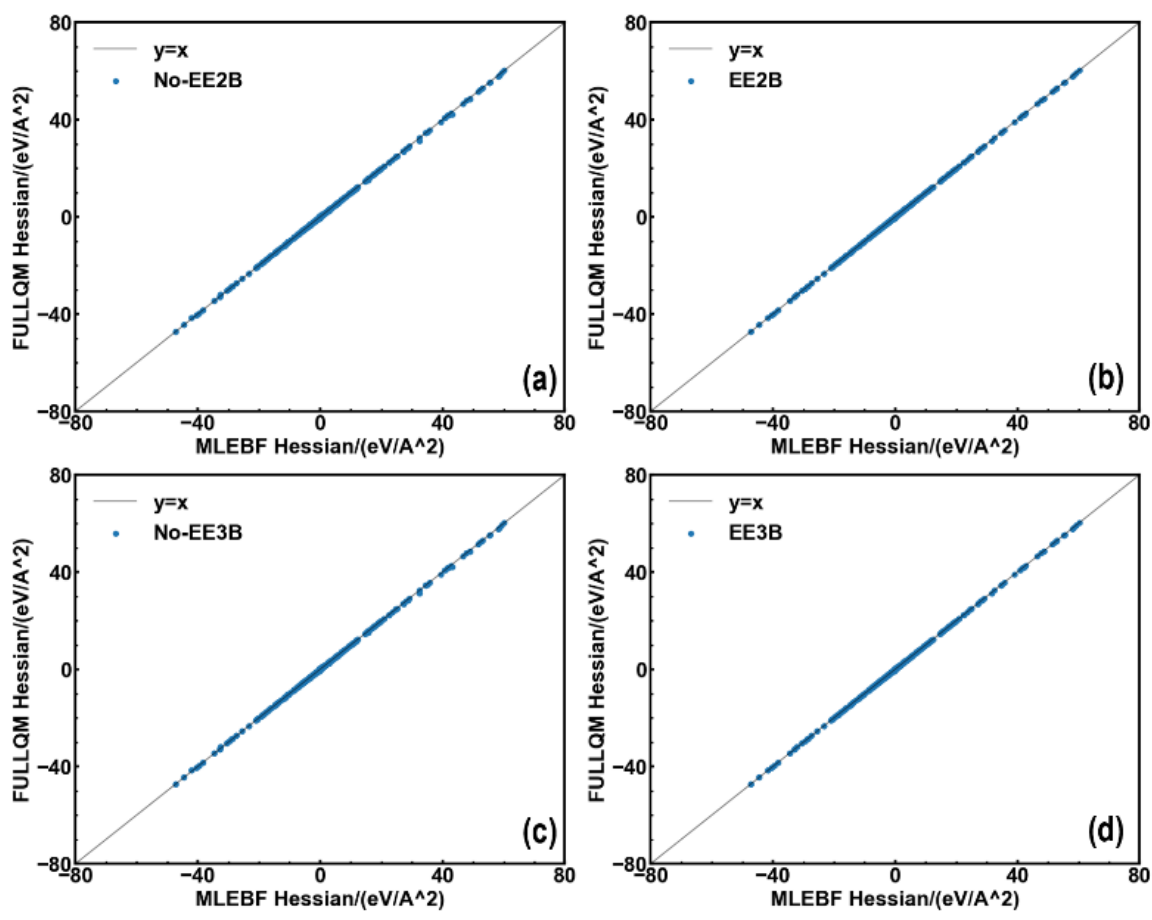

Figure S4: Comparison of full TDDFT and MLEBF(TDDFT:DFT) calculated $\mathrm{S}_{1}$ hessian matrix elements (in eV/ $/ \AA^{2}$ ) at (a-b) two- and (c-d) three-body interaction levels (a and c) without and $(b$ and $d)$ with electrostatically embedding potentials.
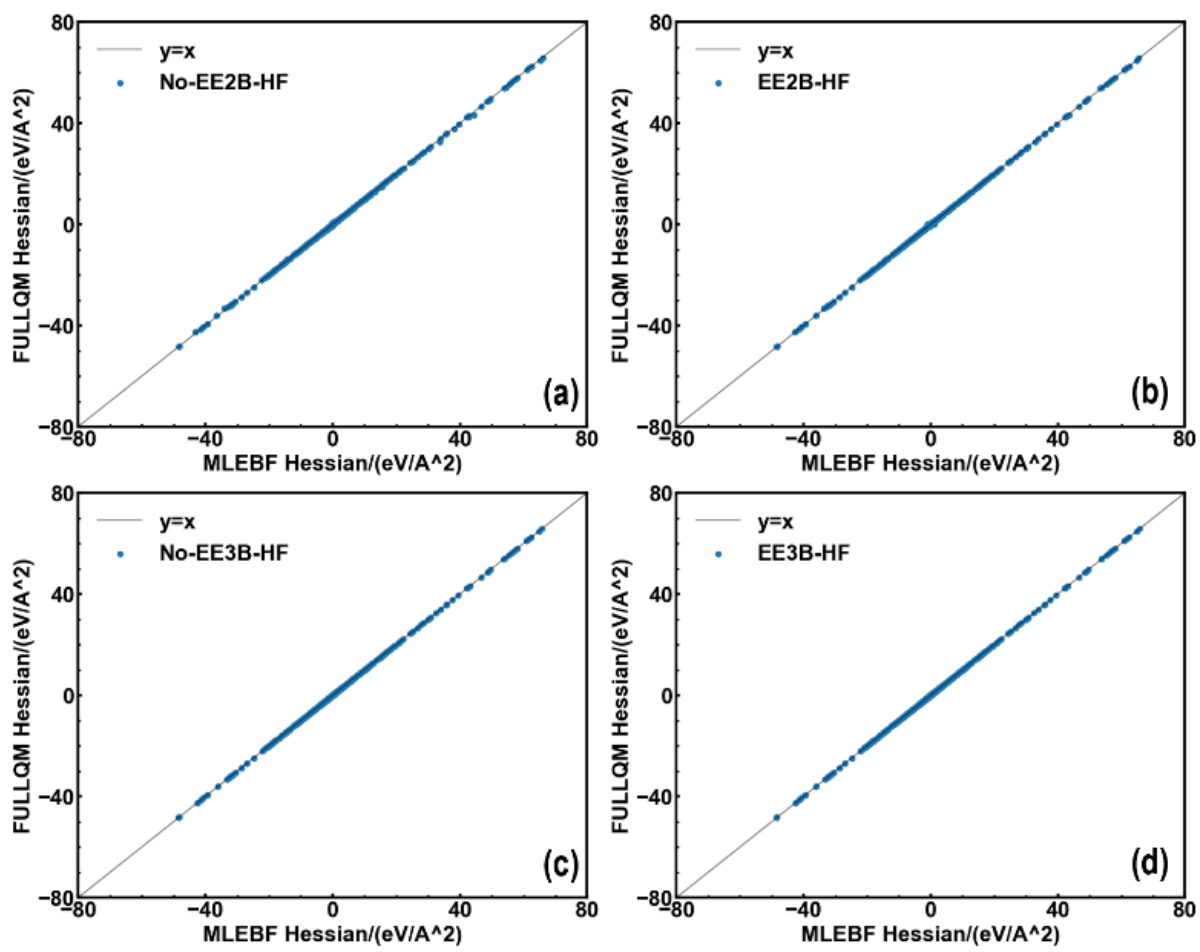

Figure S5: Comparison of full CASSCF and MLEBF(CASSCF:HF) calculated $S_{1}$ hessian matrix elements (in eV/ $/ \AA^{2}$ ) at (a-b) two- and (c-d) three-body interaction levels (a and c) without and $(b$ and $d)$ with electrostatically embedding potentials. 

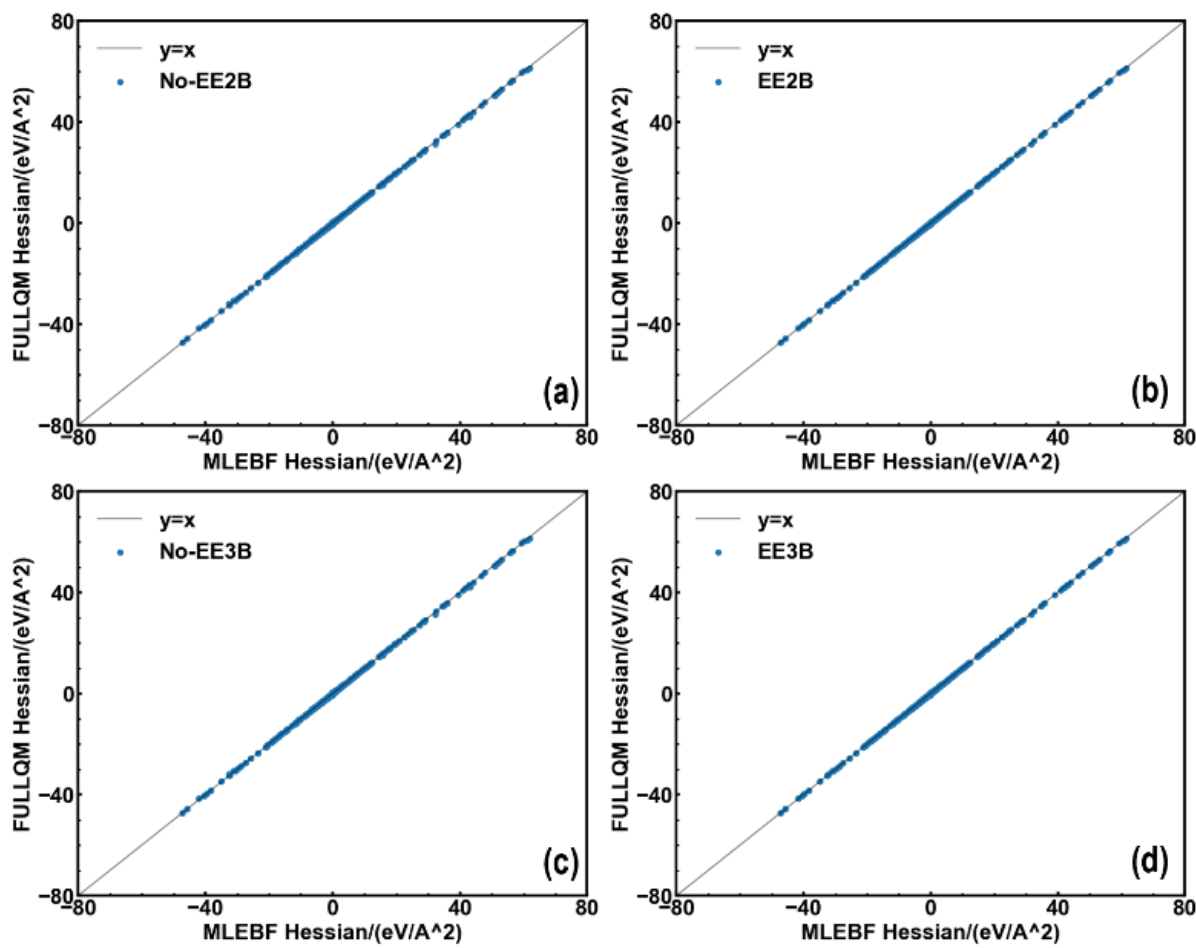

Figure S6: Comparison of full TDDFT and MLEBF(TDDFT:DFT) calculated $\mathrm{T}_{1}$ hessian matrix elements (in eV/ $/ \AA^{2}$ ) at (a-b) two- and (c-d) three-body interaction levels (a and c) without and $(b$ and $d)$ with electrostatically embedding potentials.
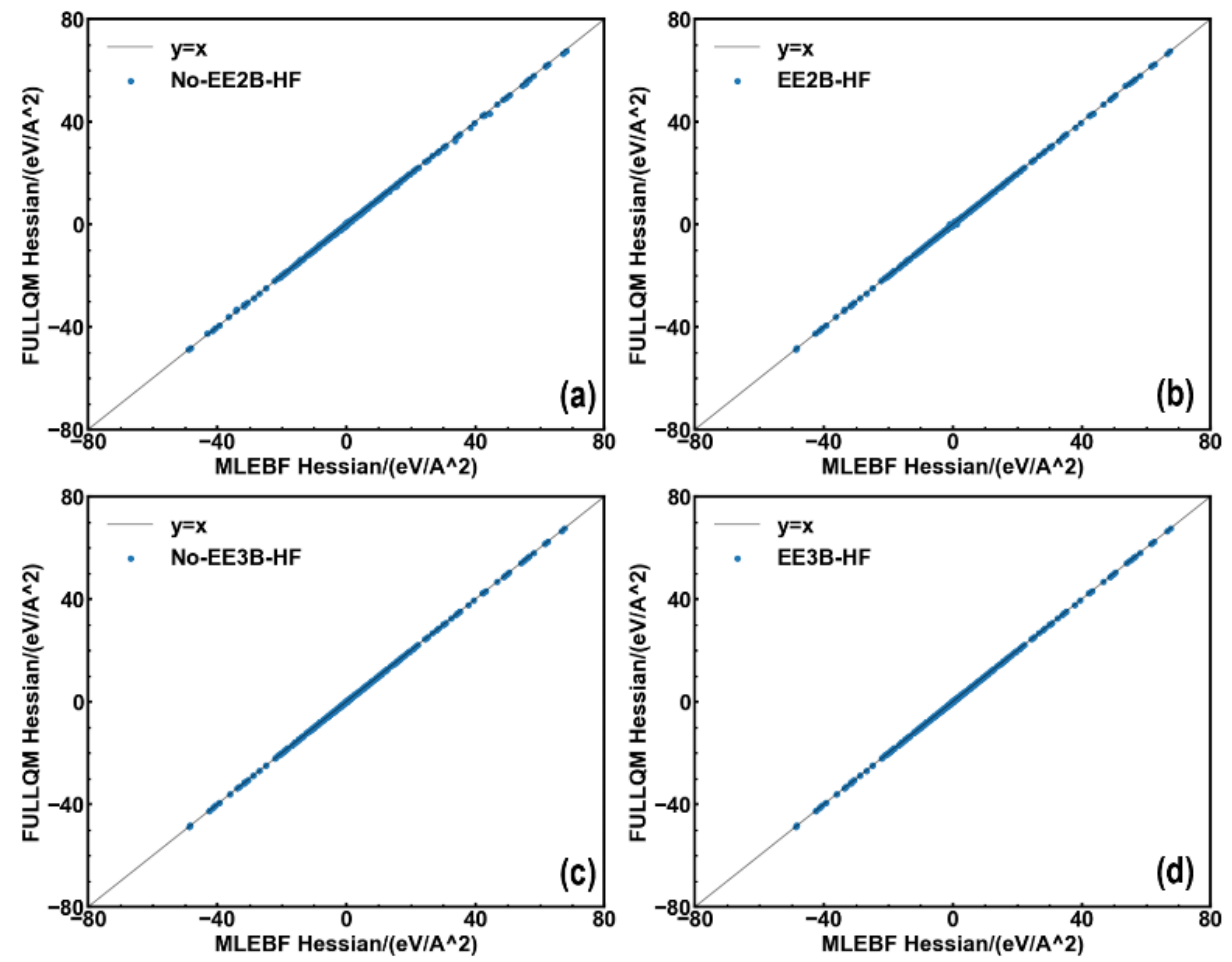

Figure S7: Comparison of full CASSCF and MLEBF(CASSCF:HF) calculated $\mathrm{T}_{1}$ hessian matrix elements (in eV/ $/ \AA^{2}$ ) at (a-b) two- and (c-d) three-body interaction levels (a and c) without and $(b$ and $d)$ with electrostatically embedding potentials. 

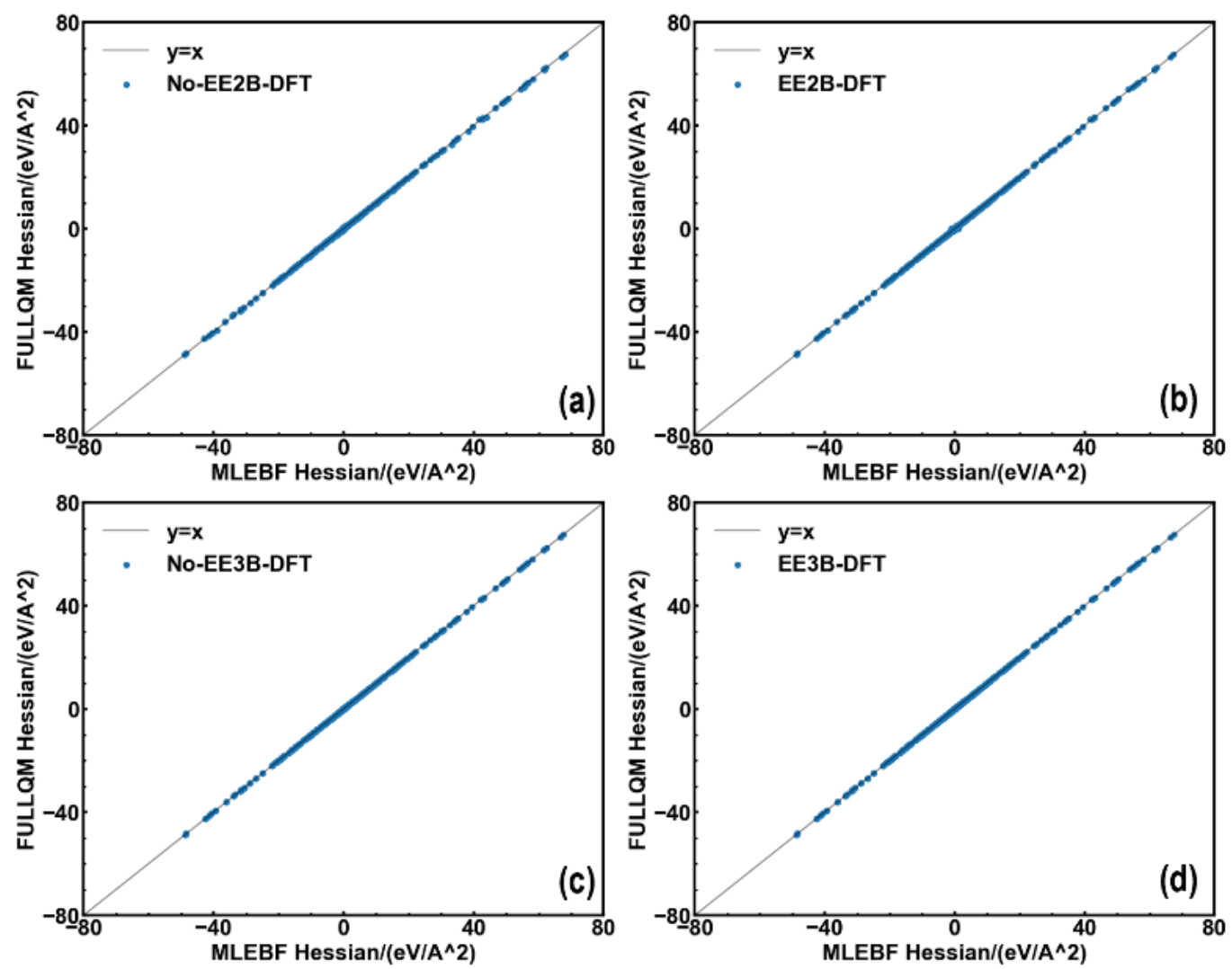

Figure S8: Comparison of full CASSCF and MLEBF(CASSCF:DFT) calculated $\mathrm{T}_{1}$ hessian matrix elements (in $\mathrm{eV} / \AA^{2}$ ) at (a-b) two- and (c-d) three-body interaction levels (a and c) without and $(b$ and $d)$ with electrostatically embedding potentials.
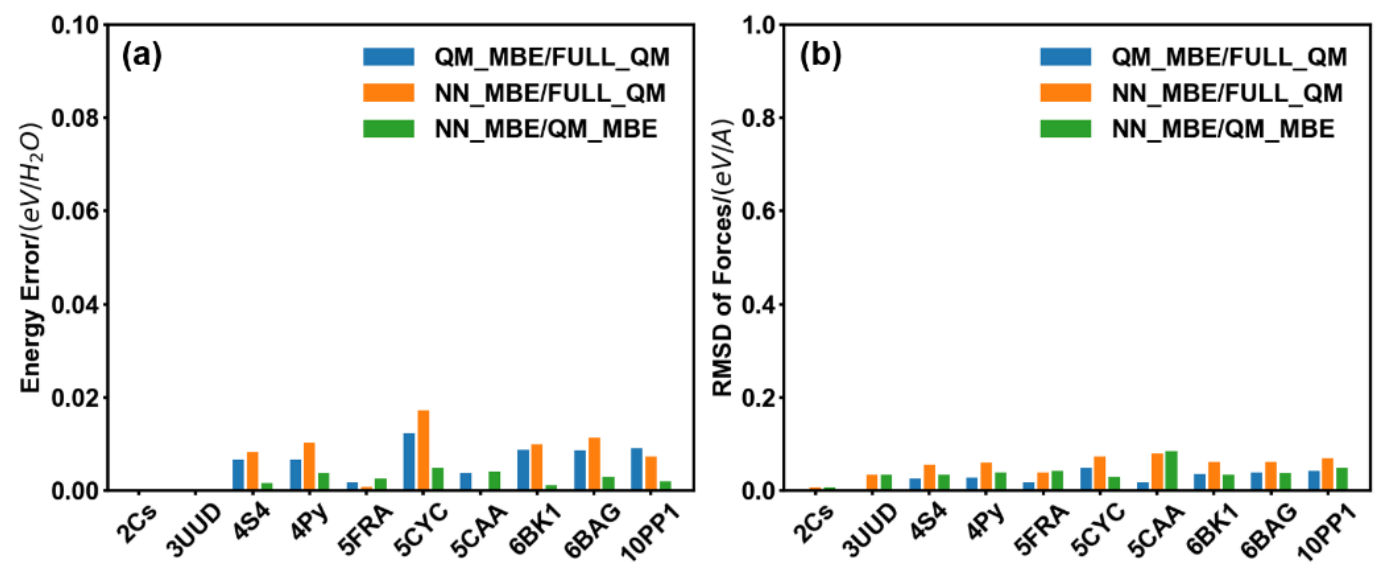

Figure S9: (a) Energy errors and (b) RMSDs of gradients of 10 selected water clusters calculated with different methods. FULL_QM stands for full DFT calculations; QM_MBE means QMbased MBE; and NN_MBE stands for ML-based MBE. 

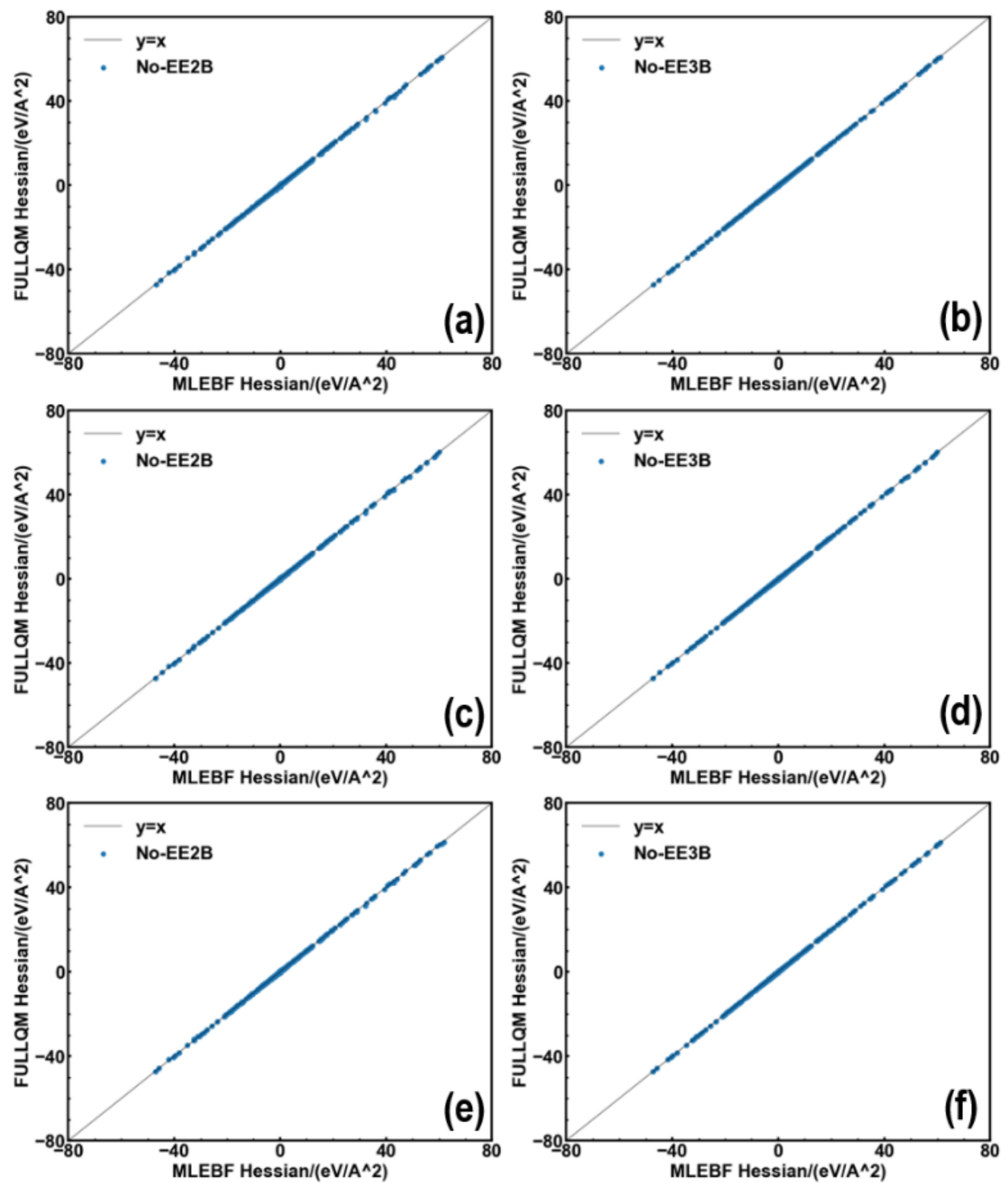

Figure S10: Comparison of ML-MLEBF(TDDFT:ML) calculated hessian matrix elements (in $\mathrm{eV} / \AA^{2}$ ) in the (a-b) $S_{0}$, (c-d) $S_{1}$, and (e-f) $T_{1}$ states compared with ab initio data. MLMLEBF(TDDFT:ML) is actually MLEBF(DFT:ML) for the $S_{0}$ state. 

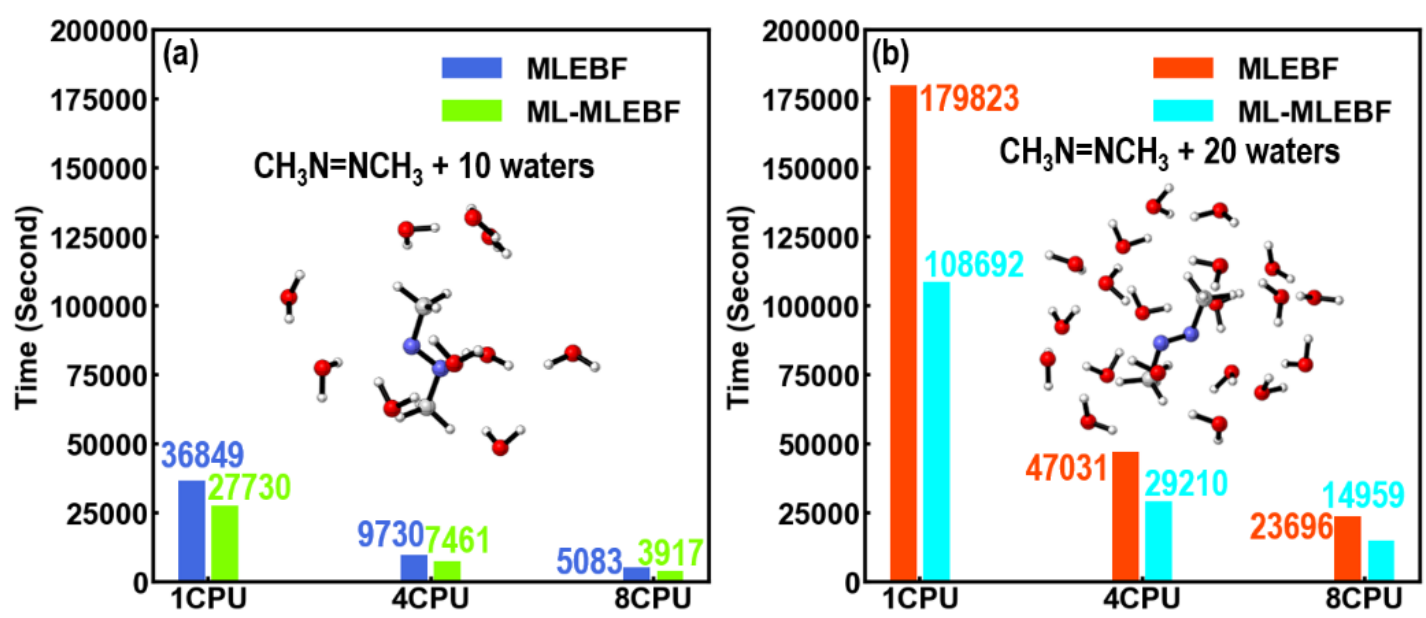

Figure S11: Comparison of CPU times of MLEBF(CASSCF:DFT) and ML-MLEBF(CASSCF:ML) calculations with three-body interaction for the $\mathrm{S}_{1}$ hessian matrices (in $\mathrm{eV} / \AA^{2}$ ) of $\mathrm{CH}_{3} \mathrm{~N}=\mathrm{NCH}_{3}$ with (a) 10 and (b) 20 water molecules.
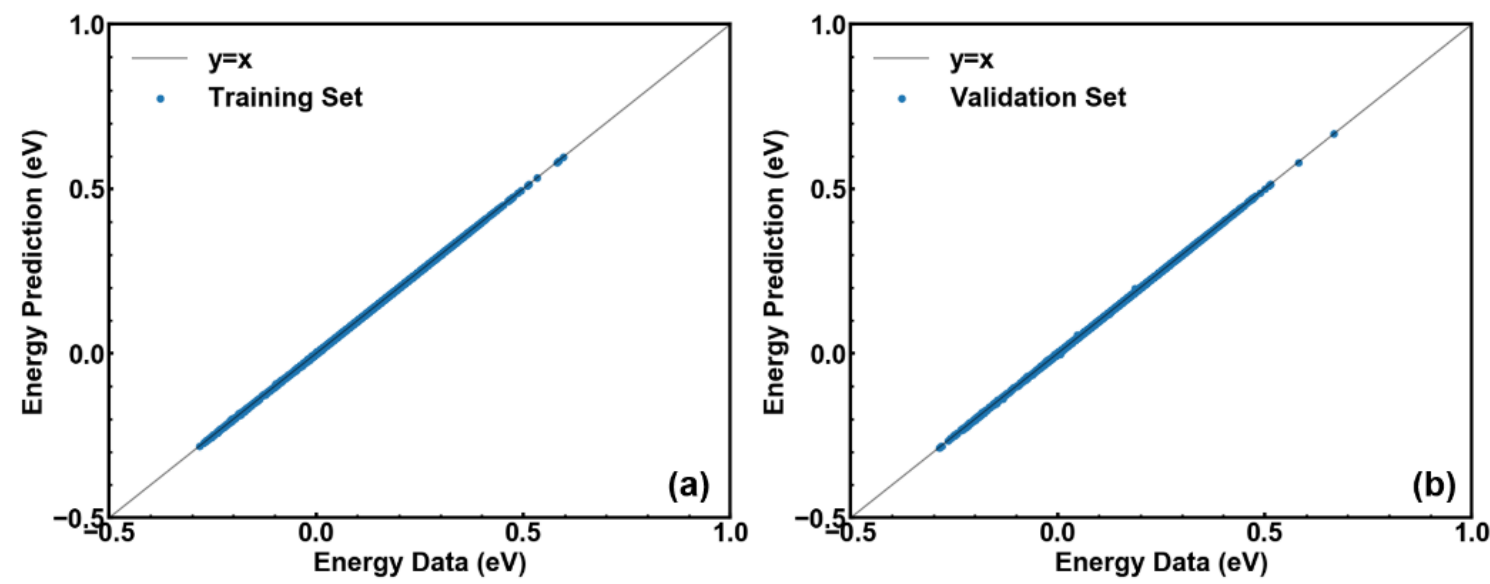

Figure S12: Comparison of ML calculated energies (in $\mathrm{eV}$ ) compared with ab initio data for both (a) training and (b) validation data.
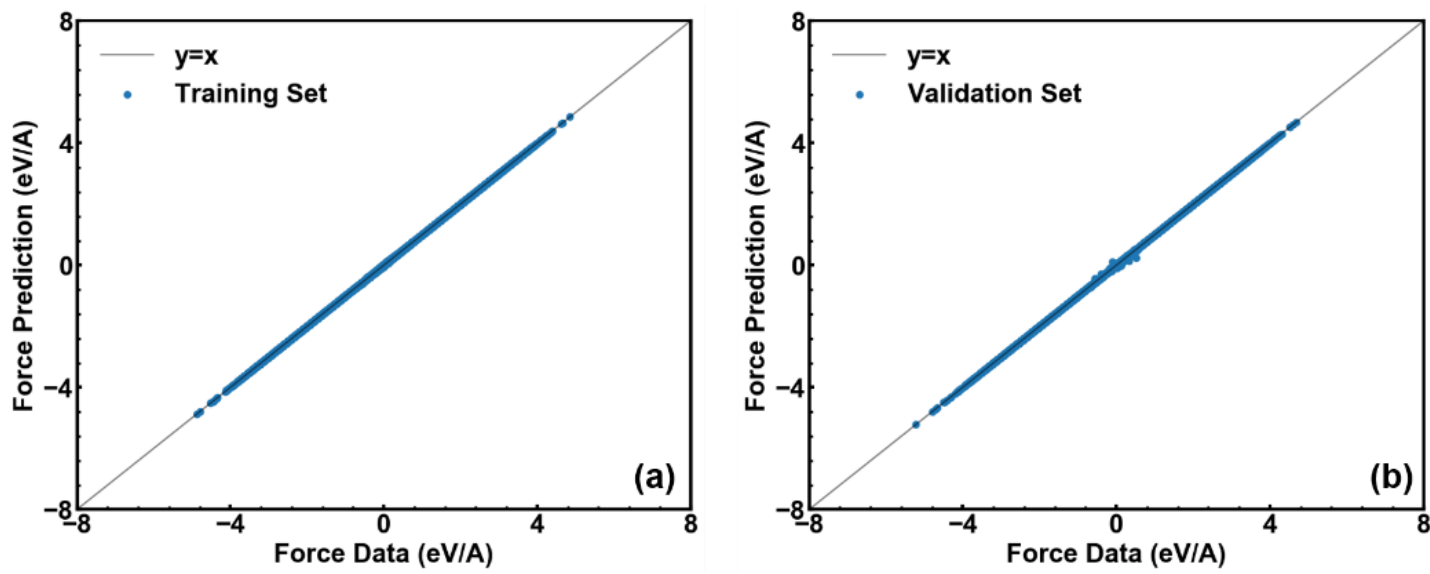

Figure S13: Comparison of ML calculated forces (in $\mathrm{eV} / \mathrm{A}$ ) compared with ab initio data for both (a) training and (b) validation data. 


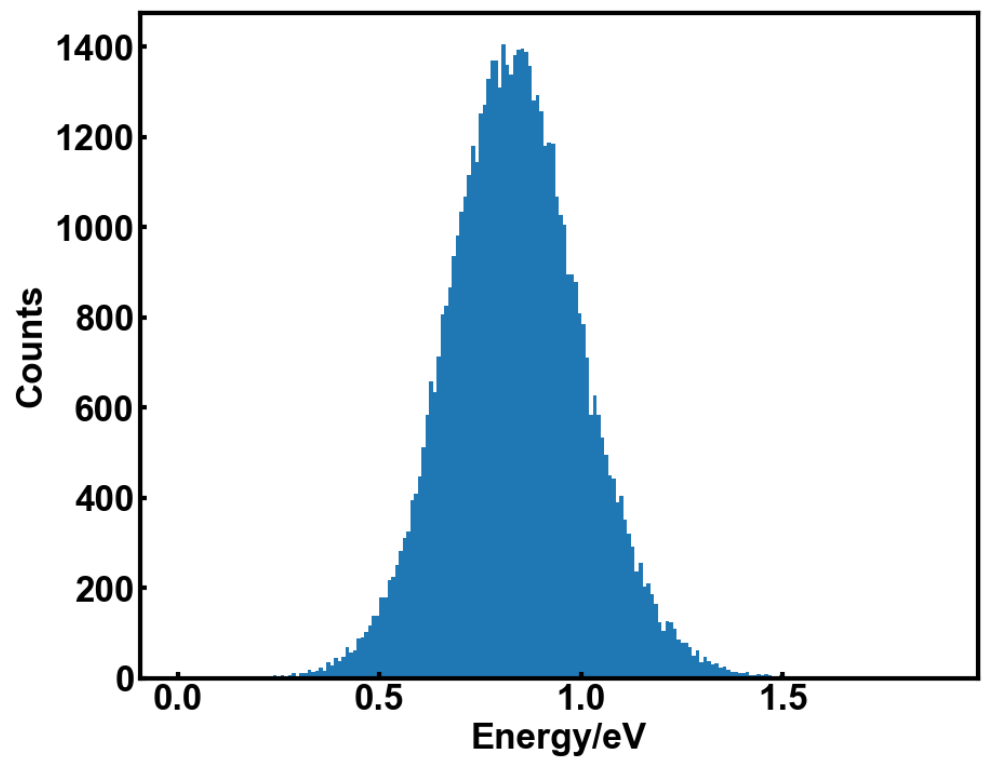

Figure S14: Energy distribution of sampling data used in neural network models' training. 